\title{
Exploring the fetal brain: is MRI always better than ultrasound?
}

\author{
Michal Lipa, Przemyslaw Kosinski, Miroslaw Wielgos
}

The $1^{\text {st }}$ Department of Obstetrics and Gynecology, Medical University of Warsaw, Poland

In many cases of suspected fetal brain abnormalities physicians refer patients for MRI scans to gather more clinical information. Usually this technique is significantly helpful, however in some cases the results may be confusing or even misleading. Despite continuous progress in the field of prenatal ultrasonography, both differential diagnosis and the nomenclature of posterior fossa defects remain challenging. The Dandy-Walker complex is commonly suspected in cases of posterior fossa abnormality or an unusual vermian appearance. However, an upward rotation and allegedly reduced dimension of the cerebellar vermis may be caused by the delayed perforation of Blake's pouch cyst and may occur in an otherwise normal fetal brain. During a routine scan for anomalies, it was therefore possible to visualize the posterior fossa clearly suggesting agenesis of the vermis (Fig. 1). This finding was also confirmed by an experienced reference center sonographer. The patient was then referred for a fetal MRI scan to either confirm or exclude the diagnosis. The MRI was performed at 28 weeks gestation, and the result confirmed the suspicions raised by the ultrasonogram (Fig. 2). The patient was subsequently counselled and provided with information on vermian agenesis. A follow-up scan at 31 weeks gestation revealed normal posterior fossa structures with a clearly present cerebellar vermis and a normal cisterna magna (Fig. 3). The baby was delivered at term, and three months later examined by a pediatric neurologist. The examination revealed a normal neurodevelopment and therefore the quality of life was expected to be normal. To date, only a very few cases of the spontaneous resolution of Blake's Pouch cyst have been reported in the literature. Despite the growing availability of fetal brain MRI scans, it must be emphasized, that in some cases, due to the complex nature of posterior fossa abnormalities, an MRI will not always improve the diagnostic process. In most cases, an ultrasound scan is enough to confirm a diagnosis, but an MRI can still be helpful in cases of poor visualization and with obese patients. Therefore, it is possible that despite the whole process of prenatal abnormality detection, parent counseling, prognosis and additional diagnostic tests being conducted by experienced sonographers using fetal MRI examinations, the initial diagnosis may still be wrong. This confirms that differential diagnosis of the posterior fossa defects may be extremely difficult and sometimes neither an ultrasound nor an MRI can guarantee an unequivocal diagnosis. If there is any suspicion of a posterior fossa defect, a multidisciplinary examination should be introduced. In such cases, careful assessment of the brainstem-vermis and brainstem-tentorium angles may help in forming a prenatal diagnosis. Values below 30 degrees suggest Blake's pouch cyst, while those above 45 degrees point to the Dandy-Walker malformation.

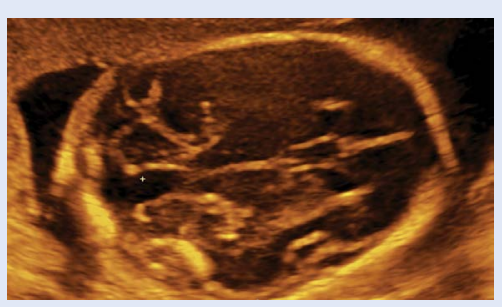

Figure 1. Absent vermis with enlarged cisterna magna at anomaly scan ( 20 weeks of gestation)

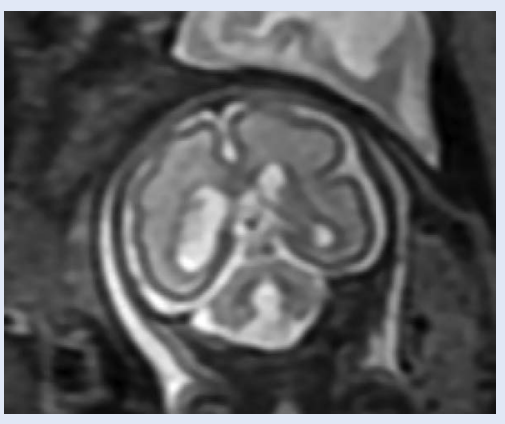

Figure 2. MRI examination at 28 weeks pointing to vermian agenesis

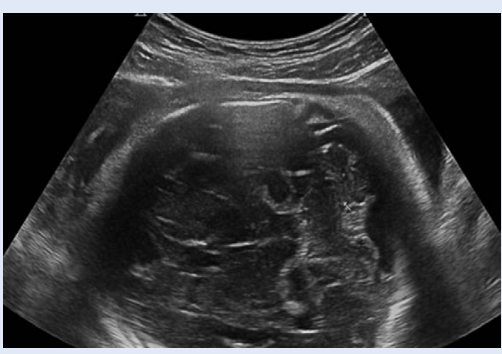

Figure 3. Normal posterior fossa appearance in an ultrasound scan at 31 weeks. Vermis, fourth ventricle and cisterna magna have normal morphology

Corresponding author:

Michal Lipa

The $1^{\text {st }}$ Department of Obstetrics and Gynecology, Medical University of Warsaw, Poland

e-mail: michallipa1@gmail.com 